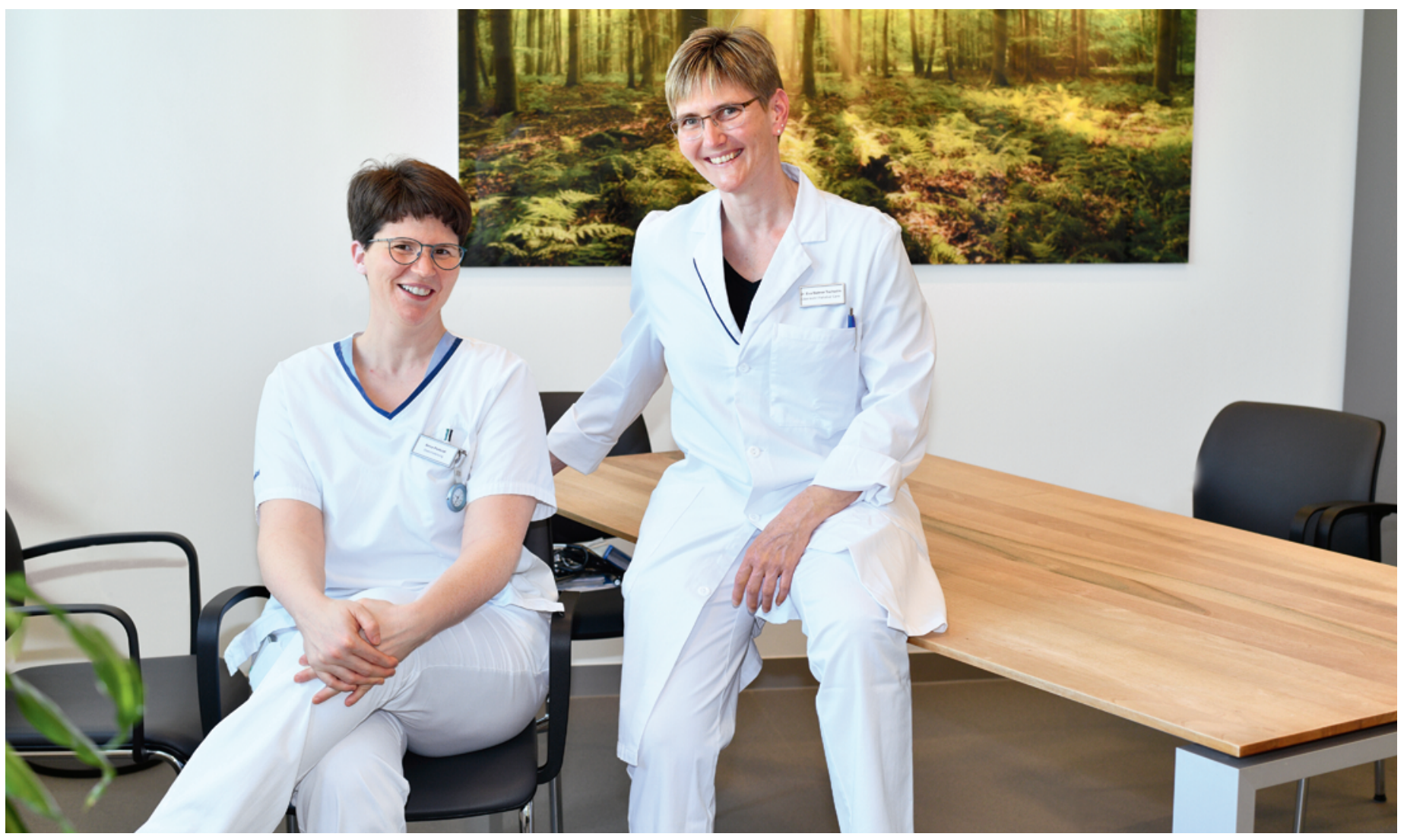

Anna Pertoldi, cheffe de l'unité de soins palliatifs (à gauche), et Eva Balmer, médecin-chef et responsable de l'unité de soins palliatifs à l'Hôpital Clara de Bâle, misent sur l'interprofessionnalité pour le traitement des personnes gravement malades.

\title{
Ensemble pour une meilleure qualité de vie
}

\section{Eva Mell}

Rédactrice en chef adjointe du Bulletin des médecins suisses

Différentes professions travaillent en étroite collaboration dans l'unité de soins palliatifs de l'Hôpital Clara à Bâle afin de soulager les symptômes des malades et de maintenir la meilleure qualité de vie possible. Pourquoi l'interprofessionnalité est ici particulièrement importante et comment elle y est vécue. Reportage.

Le cancer. A un stade avancé. Dans un corps qui n'a même pas atteint la moitié de son espérance de vie statistique. Un verdict douloureux.

C'est pourquoi cette jeune femme se trouve ici, à l'unité de soins palliatifs de l'Hôpital Clara à Bâle. Le couloir de l'unité qui compte huit lits est lumineux, les murs d'un discret vert pastel et décorés de photos grand format. Un épais tronc brun foncé s'élève vers le ciel, dangereusement incliné. Menace-t-il de tomber? Une lumière scintille près de lui, semblant le soutenir avec douceur. Eva Balmer, médecin-chef et responsable de l'unité de soins palliatifs, sort d'un bureau. C'est l'heure de la réunion interprofessionnelle matinale de l'équipe principale, composée de médecins, de soignantes et de soignants.

«La patiente souffre de douleurs physiques. Se confronter à sa propre situation lui est également douloureux. Il lui faut encore du temps pour l'accepter et s'habituer à nous», explique une infirmière. L'équipe soignante a une relation particulièrement intense avec les patientes et les patients et dispose donc d'informations souvent importantes pour le traitement. «Comment va-t-elle aujourd'hui?», demande Eva Balmer, qui ne pense pas seulement à l'état physique de la patiente. 
Car la douleur qui doit être soulagée ici, dans l'unité de soins palliatifs, dépasse les seuls symptômes physiques.

\section{La douleur physique et psychique}

La douleur a de nombreux visages. Et il faut de nombreux visages pour l'affronter. «En tant que médecin, je suis spécialisée dans le traitement médicamenteux de la douleur», explique Eva Balmer au sujet de sa fonc-

\section{La douleur a de nombreux visages. Et il faut de nombreux visages pour l'affronter.}

tion au sein de la structure interprofessionnelle. En temps normal, la douleur nous protège. Elle indique un danger que le corps est sollicité, menacé ou même détruit.

Dans le meilleur des cas, les traitements médicaux qui se limitent à la douleur physique suffisent à la faire disparaître. La menace est éliminée, le problème est résolu, la douleur se dissipe. Mais avec un diagnostic de cancer mortel, c'est plus compliqué. «L'impuissance dans laquelle se trouvent les patientes, les patients et leurs proches après un diagnostic de cancer peut provoquer une douleur psychique qui se manifeste physiquement», explique David Hutter, médecin-chef à l'unité de soins palliatifs.

Il ne s'agit pas de donner plus d'importance à la douleur physique ou psychique, l'une ne prime pas sur l'autre. «Nous ne pouvons pas opposer la douleur psychique et la douleur physique», explique Andreas Dörner, responsable des services psychologiques à l'Hôpital Clara et membre de l'équipe interprofessionnelle à l'unité de soins palliatifs. En traitant la douleur psychique, il est possible de renforcer l'effet des antalgiques.

Les exemples tirés de l'éventail des traitements proposés par les services psychologiques parlent d'euxmêmes: la musicothérapie peut améliorer la respiration, ce qui permet de relâcher les tensions et d'améliorer le bien-être physique. Et tout comme l'art-thérapie, elle aide les patientes et les patients à réaliser que, même au stade terminal d'un cancer, il existe autre chose que la douleur. «Les gens réalisent qu'ils peuvent faire quelque chose ici et explorer des lieux bénéfiques en eux", explique Lucia Stäubli, art-thérapeute. Andreas Dörner le résume de la manière suivante: "La douleur est un état dans lequel je me trouve. Dès que je suis en mesure de voir d'autres états, il devient plus flexible.»

\section{La convergence des spécialités}

15 heures: la réunion interprofessionnelle hebdomadaire avec l'équipe élargie se tient dans la salle de séjour de l'unité de soins palliatifs. Ici, tous les cas sont discutés en grand groupe. En plus de l'équipe principale composée de médecins et de soignants, elle comprend également des physiothérapeutes, des membres

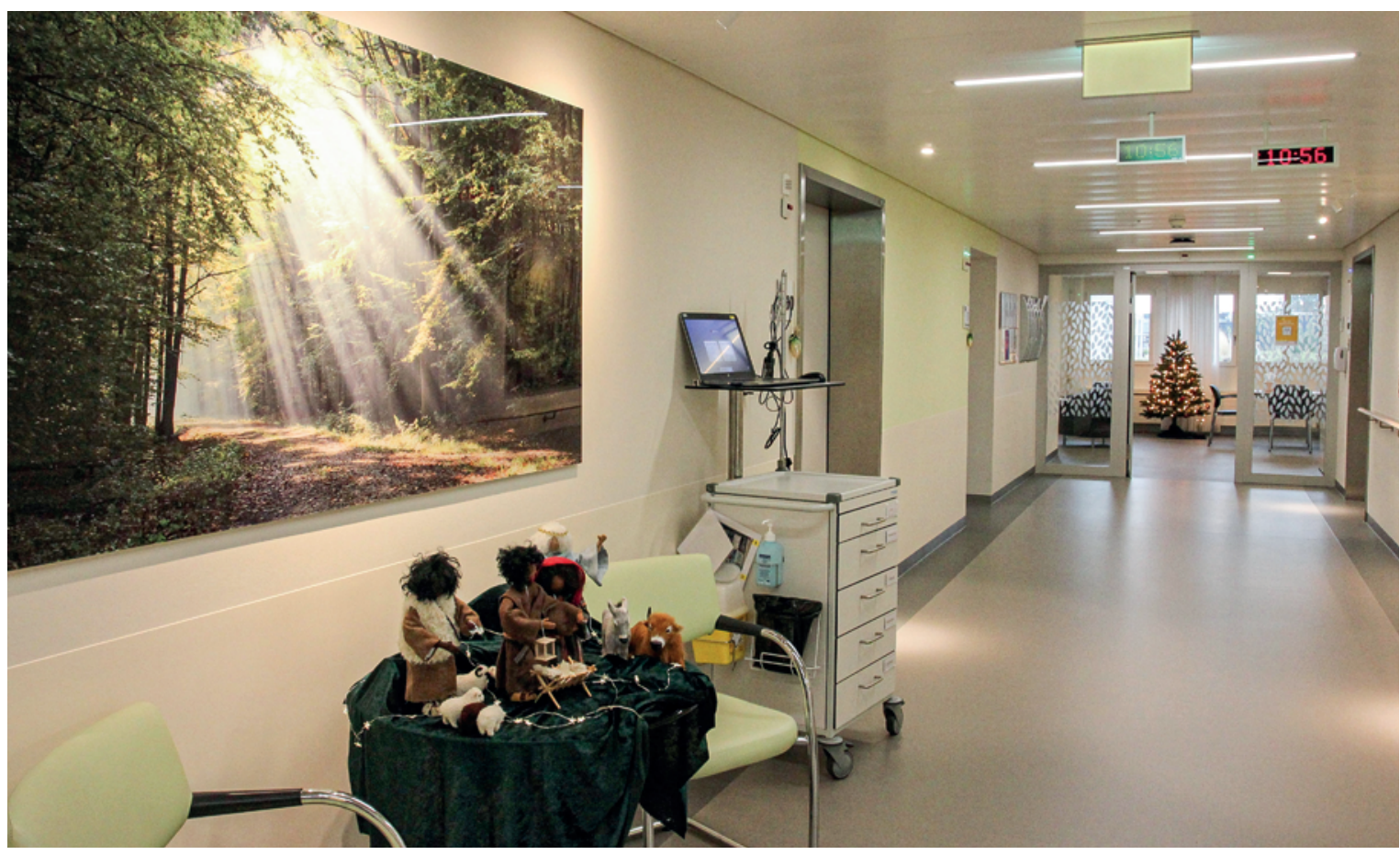

Vue sur le couloir de l'unité de soins palliatifs de I'Hôpital Clara à Bâle. 
de l'aumônerie, du Case Management et des services psychologiques. Dix personnes sont présentes ce jeudi. A côté de la table de réunion, les rayons de soleil sur la photo au mur semblent éclairer l'équipe soignante. Des troncs d'arbres étroits s'élèvent vers le ciel, leurs cimes sortent du cadre de l'image, mais le sol de la forêt est visible. Nourries par le bois et les feuilles mortes, de nouvelles plantes poussent. L'ombre ne disparaît du sol de la forêt que là où les rayons lumineux le touchent. Chaque rayon compte.

Eva Balmer regarde l'assistance et commence avec le cas suivant. La jeune patiente, dont le cancer est très avancé, souffre de douleurs très complexes. «Elle se rend compte de l'impact de la maladie sur sa vie», explique le médecin, passant ainsi aux composantes psychologiques de la douleur. Une infirmière raconte que la patiente préférerait vivre à nouveau dans son propre appartement, le physiothérapeute raconte que la jeune femme aimerait gagner en mobilité et en force et faire des exercices dans les locaux de physiothérapie. «Mais

\section{«L'impuissance dans laquelle se trouve une personne diagnostiquée d'un cancer peut causer une douleur psychique ressentie phy- siquement."}

le simple fait de prendre une douche lui fait mal. Elle ne peut pas encore évaluer son état", commente une infirmière. L'art-thérapeute attire l'attention sur le fait que la relation de la patiente avec son père n'est pas encore claire. Une autre infirmière informe que la patiente a probablement aussi un frère. La responsable de gestion des cas ajoute: «La rente est réglée.» Et l'aumônière demande: «Dois-je attendre? Vous me direz si je dois venir?»

La réunion interprofessionnelle permet de réunir toutes les informations qui pourraient être utiles au traitement des patientes, des patients et de leurs proches. «Le patient ou la patiente est au centre, mais nous impliquons également l'entourage. Il est donc important de savoir si tous les proches sont informés", explique Anna Pertoldi, cheffe de l'unité de soins palliatifs.

\section{Série sur l'interprofessionnalité}

La collaboration entre les spécialistes de différentes professions de la santé est considérée comme un levier important afin de relever les défis du système de santé. Où en est la Suisse dans ce domaine? Quels avantages apporte l'interprofessionnalité et quelles en sont les limites? Nous éclairons le sujet sous divers angles dans une série d'articles.
Les soins palliatifs spécialisés à I'Hôpital

\section{Clara}

L'unité de soins palliatifs de I'Hôpital Clara est un service autonome de huit lits dédiés aux «soins palliatifs spécialisés». Elle accueille des patientes et des patients atteints de maladies incurables et mortelles dans des situations pathologiques complexes ou instables. A côté des douleurs physiques sont aussi pris en compte les aspects psychologiques, sociaux et spirituels. L'équipe interprofessionnelle principale se compose $d$ 'infirmières et de médecins ayant des compétences spécialisées en soins palliatifs. L'équipe élargie comprend des professionnels de la psychologie, de la musico- et de l'art-thérapie, de l'aumônerie, du travail social, de la physiothérapie et de la thérapie nutritionnelle.

Chaque pièce du puzzle est essentielle pour traiter de manière globale la douleur des personnes concernées et de leurs proches. «Face aux médecins, les patientes et les patients veulent souvent se montrer forts. Auprès de l'équipe soignante, ils recherchent davantage du réconfort», explique le psychologue Andreas Dörner. Les malades rapportent au physiothérapeute leurs objectifs physiques qu'ils ne discuteraient pas avec des personnes d'autres professions. "Lors de la séance interprofessionnelle, nous réunissons toutes ces informations», explique Andreas Dörner, qui apprécie le respect dont jouissent tous les corps de métier à l'unité des soins palliatifs: «Nous savons comment utiliser les forces des différentes professions.»

\section{Chaque profession a ses spécialités}

C'est justement ce qui importe à Eva Balmer: «Nous devons sans cesse redéfinir de quelles connaissances spécialisées une patiente ou un patient peut profiter.» En tant que responsable de l'unité de soins palliatifs, elle se charge de la cohésion de l'équipe et collecte les informations. Mais: «Aucun d'entre nous n'a plus d'importance qu'une autre personne, chacun a ses points forts.»

La réunion est terminée, le soleil se couche dehors. Il continue à briller sur les photos des murs de l'unité de soins palliatifs. Sa lumière tombe sur un chemin forestier, chaque rayon devient important à un endroit différent pour que le chemin puisse être parcouru en toute sécurité. Dans le couloir, Eva Balmer et Anna Pertoldi échangent à voix basse avant de continuer à travailler, à affronter la douleur, chacune à sa manière et pourtant ensemble.

\section{Crédits photos}

Photo page 1: aimablement mise à disposition par l'Hôpital Clara Photo page 2: Eva Mell 